\title{
Potential field based optimization of a prey-predator multi-agent system
}

\author{
Valentin Baillard* Alexandre Goy* Nicolas Vasselin* \\ Cristina Stoica Maniu** \\ * CentraleSupélec, LMI department, Gif-sur-Yvette, France (e-mail: \\ \{valentin.baillard; alexandre.goy; nicolas.vasselin\}@student.ecp.fr). \\ ** Laboratoire des Signaux et Systèmes, CentraleSupélec-CNRS-Univ. \\ Paris-Sud, Université Paris Saclay, Gif-sur-Yvette, France (e-mail: \\ cristina.maniu@centralesupelec.fr)
}

Keywords: Multi-agent systems, potential field, numerical simulation, optimization.

\section{INTRODUCTION}

The field of multi-agents systems (MAS) is marked out by various methods and approaches (Ren and Cao (2011)). For formation control, graph theory (Mesbahi and Egerstedt $(2010)$ ) is a natural paradigm for systems with imperfect information coupled to agents' dynamics, while set-theoretic methods (Blanchini and Miani (2007)) are suitable for addressing uncertainty and implementing constrained control for MAS (Nguyen (2016)). Intelligent control, including Artificial Intelligence (AI) and game theory (Vrancx et al. (2007)) capabilities, is a way to investigate individual behavior effects on collective processes. Potential fields (Leonard and Fiorelli (2001)) are used for formation tracking (Ren and Cao (2011)), but scarcely used to the best of the authors' knowledge for formation producing.

In this context, this work is part of an educational project on multi-agent systems for the analysis of the dynamics of a swarm of mobile agents. This paper proposes a framework for the optimization of adversarial potentialbased prey-predator-like problems. The adversarial potentials are decomposed onto a basis set with different weights. Each weight is individually optimized in a Particle Swarm Optimization-like manner using a Covariance Matrix Adaptation Evolution Strategy (CMA-ES). The first contribution of this paper is related to the partitioning of the potentials followed by a global cost function optimization step, allowing for a topology-based, parameter-based or constraint-based behavioral analysis of a MAS, in a problem with multiple variables. The second contribution is a proof of concept on a "Cops \& Robbers" case study, relying both on optimization and environmental variables.

The paper is organized as follows. Section 2 introduces the mathematical tools and methods. Section 3 presents the considered scenario and assumptions, as well as an analysis of the simulation results. Concluding results and current work are drawn in Section 4.

Notation. Let $x, y \in \mathbb{R}^{n}$. Their euclidean distance is denoted by $d(x, y)$ and the $i$-th component of $x$ by $x^{(i)}$.

\footnotetext{
1 The authors acknowledge the support of the Research Professions stream (Filière Métiers de la Recherche) of CentraleSupélec.
}

\section{THEORETICAL BACKGROUND}

The general framework of this paper considers interactions between two adversarial teams of agents (team $\mathcal{A}$ and team $\mathcal{B})$ evolving in an environment $\mathcal{E} \subseteq \mathbb{R}^{n}$, at speed $v_{\mathcal{A}}$ and $v_{\mathcal{B}}$ resp., along with a set of objectives $\mathcal{O}$. With respect to a given objective function $F$, team $\mathcal{A}$ minimizes $F$ and team $\mathcal{B}$ minimizes the opposite objective function $-F$. The behavior of both teams is achieved by optimizing a potential field decomposed according to its sources.

\subsection{Potential fields}

A potential is a differentiable function $U: \mathcal{E} \rightarrow \mathbb{R}$. Let $c \in \mathcal{A} \cup \mathcal{B}$ be an agent. It generates a potential $U_{c}^{\mathcal{A}}$ towards team $\mathcal{A}$ and a potential $U_{c}^{\mathcal{B}}$ towards team $\mathcal{B}$. For any objective $o \in \mathcal{O}$ there are similar potentials $U_{o}^{\mathcal{A}}, U_{o}^{\mathcal{B}}$. Moreover, the borders of the ambient environment generate additional potentials $U_{\mathcal{E}}^{\mathcal{A}}$ and $U_{\mathcal{E}}^{\mathcal{B}}$. By the principle of superposition, any agent $c \in \mathcal{C}$ (where $\mathcal{C}$ is either $\mathcal{A}$ or $\mathcal{B}$ ) is thus subject to a potential of the form

$$
U_{c}=\sum_{a \in \mathcal{A}} U_{a}^{\mathcal{C}}+\sum_{b \in \mathcal{B}} U_{b}^{\mathcal{C}}+\sum_{o \in \mathcal{O}} U_{o}^{\mathcal{C}}+U_{\mathcal{E}}^{\mathcal{C}}
$$

In order to restrict the dimensionality of the problem, only potentials of the following shape are allowed

$$
U: x \mapsto \sum_{k \in \mathbb{Z}} u_{k} \cdot d(x, y)^{k}, \text { with } y \in \mathcal{E}
$$

where $\left(u_{k}\right)_{k \in \mathbb{Z}}$ is a finitely supported real sequence of optimization parameters. The environment potential takes into account the distance from $x$ to the borders of the environment (considered as a hyperrectangle parallel to the $x, y$ axes). In this setting, the environment potential is a finite sum of terms of the shape

$$
U: x \mapsto \sum_{k \in \mathbb{Z}} u_{k}^{(i)}\left|x^{(i)}-\alpha\right|^{k}
$$

for $i \in \llbracket 1, n \rrbracket$ and some $\alpha \in \mathbb{R}$. The system starts from arbitrary initial positions for the teams $\mathcal{A}$ and $\mathcal{B}$ and evolves in a discrete time. At time $t+1$, each agent $c \in \mathcal{C}$ (with $\mathcal{C}$ either $\mathcal{A}$ or $\mathcal{B}$ ) is moving in the opposite direction of the gradient $-\nabla U_{c}$ at speed $v_{\mathcal{C}}$, with $U_{c}$ computed at time $t$. After a specified number of steps, or when it meets a stopping criterion, the value of $F$ is returned. 


\subsection{Parameters optimization}

For the proposed model, all the behavioral information is contained into the parameters $u_{k}$ defined in $(2)$ and $u_{k}^{(i)}$ defined in (3). A CMA-ES algorithm (Hansen (2007)) is used to optimize the parameters regarding the objective function $F$. CMA-ES is a stochastic optimization strategy best applied to real functions of which only evaluations are known (e.g. simulation results).

\section{CASE STUDY}

This section presents numerical simulations in a specific case based on a true story (Williams (2015)). This case study (Fig. 1) considers $n_{\mathcal{A}}$ policemen drones (light blue dots) and $n_{\mathcal{B}}$ yakuzas drones (green dots), moving with equal speeds in a rectangle defined by the 2 dark blue dots. The yakuzas have 2 physical objectives (yellow dots): one supply objective $s$ (center) and 4 delivery objectives $d$ (sides). A delivery is a completion from $s$ to $d$. Initially, each yakuza seeks out objectives $d$. If it reaches a $d$, the new objective becomes $s$. If it reaches $s$, it seeks objectives $d$ again. A yakuza is removed from the simulation if it encounters a policeman. Any drone colliding with a rectangle border is also removed. The objective function $F$ is the total number of deliveries made by the yakuzas during the simulation.

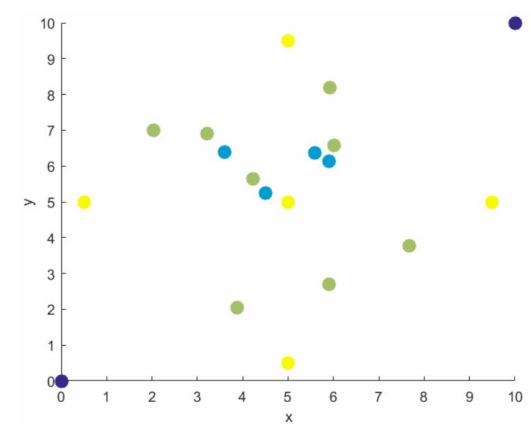

Fig. 1. Extract of a Matlab simulation $\left(n_{\mathcal{A}}=4, n_{\mathcal{B}}=8\right)$

Policemen have no information about the yakuzas' objectives. The potentials are decomposed on the basis described in (1) and (3) restricted to the powers $k \in$ $\{-2,-1\}$ for computational reasons. This leads to 14 parameters entering the CMA-ES algorithm, which are then optimized regarding a single team's objective function. A video illustration of the simulation results is provided at www.youtube.com/watch?v=GscU1e3sc04.

Repulsive interaction corresponds to $u_{k}>0$, while $u_{k}<0$ yields an attractive interaction. The potential coefficients corresponding to $k=-1$ are associated to a long-range interaction. The long-range attractive potential of the objectives is observed to be larger than the other longrange potentials. The $k=-2$ coefficients (corresponding to a short-range action) are displayed in Fig 2, for a number of policemen $n_{\mathcal{A}}=4$. Each plot represents the $u_{k}$ parameter of a component of the potential $U_{\mathcal{B}}$, according to (1)-(3). Three phases are identified. After a highly non-beneficial situation for a single yakuza, the behavior becomes strongly objective-oriented for $n_{\mathcal{B}} \geqslant 2$ (i.e. $u_{-2}$ of the objectives goes to the normalized value -1 in Fig.
2). A drastic behavior change can be seen from $n_{\mathcal{B}} \approx 6$ : for more yakuzas, the winning strategy goes from full to mitigated inter-yakuza and yakuza-policemen repulsion (the blue and orange curves scale down towards 0 in Fig. 2). This can be understood as a trade-off between safety at low $n_{\mathcal{B}}$ and high-pay risk from collective action inducing easier workaround at higher $n_{\mathcal{B}}$. The borders intuitively remain strongly repulsive all along.

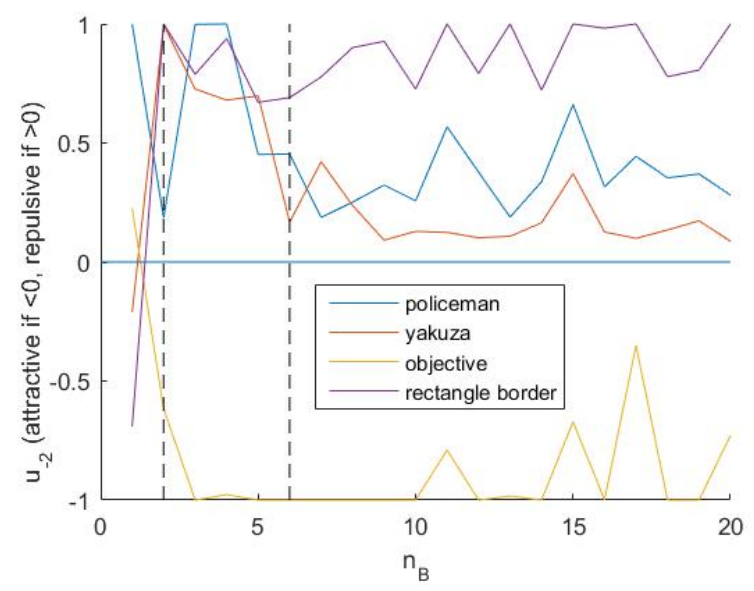

Fig. 2. Team $\mathcal{B}$ potential coefficients $u_{k}$ w.r.t. $n_{\mathcal{B}}$ yakuzas

\section{CONCLUSION AND FUTURE WORK}

This paper proposed a potential-based framework using a decomposition basis for the optimization of the behaviors in a prey-predator-like problem. The simulation results highlighted a change of strategy for different numbers of agents in a "Cops \& Robbers" scenario.

Future investigations on the $F\left(n_{\mathcal{A}}, n_{\mathcal{B}}\right)$ map will define the advantage regions for each team. Analyzing the optimized behaviors from a refined model could unveil new strategies and confirm existing ones: by allowing new environment topologies (e.g. objectives numbers and locations, obstacles), by allowing lower $k$-values or by implementing adversarial optimization.

\section{REFERENCES}

Blanchini, F. and Miani, S. (2007). Set-theoretic methods in control. Birkhäuser.

Hansen, N. (2007). The CMA evolution strategy: a comparing review. Towards a new evolutionary computation. Studies in fuzziness and soft computing, 192.

Leonard, N.E. and Fiorelli, E. (2001). Virtual leaders, artificial potentials and coordinated control of groups. Proc. of the 40th IEEE CDC.

Mesbahi, M. and Egerstedt, M. (2010). Graph theoretic methods in Multiagent networks. Princeton Univ. Press.

Nguyen, M.T. (2016). Safe predictive control for multiagent dynamical systems. Ph.D. thesis, CentraleSupélec.

Ren, W. and Cao, Y. (2011). Distributed coordination of Multi-Agent networks. Springer.

Vrancx, P., Verbeek, K., and Nowé, A. (2007). Decentralized learning in Markov games. IEEE Transactions on Systems, Man, and Cybernetics.

Williams, R. (2015). Tokyo police are using drones with nets to catch other drones. The Telegraph. 